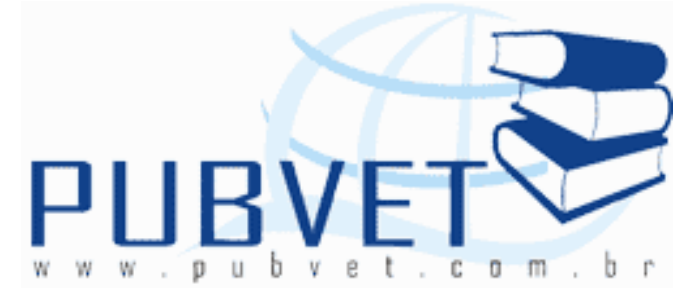

PUBVET, Publicações em Medicina Veterinária e Zootecnia.

\title{
Acidente vascular cerebral em cão - Relato de caso
}

Débora Polleto Valente, Lucas Pimentel Cardoso, Alysson Rodrigo Lamounier, Luiz Fernando Lucas Ferreira

\section{Resumo}

Um cão macho, pastor alemão, com 14 anos, foi atendido no CECCA - PUC Betim (Clínica e Cirurgia de Animais), apresentando prostração, redução de apetite, e em decúbito lateral. O exame clínico revelou hipertermia, desidratação acentuada, taquicardia, estertor úmido, presença de secreção bilateral nas narinas, caquexia e reflexos dos membros posteriores aumentados. Apartir dos sinais clínicos foi suspeitado: Acidente Vascular Cerebral, Cinomose e Tumor Cerebral. O acidente vascular cerebral (AVC) é uma doença rara em cães e geralmente é causada por uma isquemia ou hemorragias dentro do parênquima cerebral. $O$ diagnóstico se baseia primeiramente nos sinais neurológicos, delírio, coma, paralisia uni ou bilateral, sendo confirmado através de tomografia computadorizada e ressonância magnética, ou somente de diagnóstico histológico e necropsia. Tratamento inclui a prevenção a doenças cardiovasculares e metabólicas, e após o quadro instalado, pode se fazer o uso de trombolíticos, vasodilatadores cerebrais, corticóides e vitaminas do complexo B. Conclui se que a histopatológica é fundamental para diagnostico diferencial de cinomose, neoplasias cerebral.

Palavras-Chave: Cão, AVC, Tumor Cerebral 


\section{Cerebrovascular accident in dogs - Case report}

\section{Abstract}

A male dog, German shepherd, aged 14, was treated at the CECCA - PUC Betim (Animal Clinic and Surgery), with prostration, decreased appetite, and in lateral recumbency. Clinical examination revealed hyperthermia, severe dehydration, tachycardia, moist rattle, secretion bilateral nostrils, cachexia, and increased reflexes in the hindlimbs. Starting clinical signs was suspected: Stroke, Distemper and Tumor Cerebral. The cerebrovascular accident (CVA) is a rare disease in dogs and is usually caused by ischemia or bleeding within the brain parenchyma. The diagnosis is based primarily on neurological signs, delirium, coma, paralysis, unilateral or bilateral, was confirmed by computed tomography and magnetic resonance imaging, or only a tissue diagnosis and autopsy. Treatment includes prevention of cardiovascular and metabolic diseases, and after the framework installed, you can make use of thrombolytics, vasodilators, cerebral, steroids and vitamin B complex Concluded that the histopathological differential diagnosis is essential for distemper, brain neoplasms

Keywords: Dog, CVA, stroke, Cerebral Tumor

\section{Introdução}

O acidente vascular cerebral (AVC) é uma patologia originada por lesões que afetam os vasos sanguíneos cerebrais e pode ser dividida em duas categorias: AVC isquêmico e AVC hemorrágico. Na maioria dos casos, o AVC tem como causas diabetes, hipertensão, insuficiência renal crônica e hiperadrenocorticismo, não tendo predisposição racial, mas a senilidade é um indício ${ }^{3,10}$.

O AVC isquêmico pode ser resultante da oclusão de vasos sanguíneos cerebrais, vindo de trombos e embolismos, privando o cérebro de oxigênio e glicose. O AVC hemorrágico é resultante da ruptura da parede do vaso 
VALENTE, D.P. et al. Acidente vascular cerebral em cão - Relato de caso. PUBVET, Londrina, V. 6, N. 25, Ed. 212, Art. 1412, 2012.

sanguíneo dentro do parênquima cerebral ou do espaço subaracnóideo, causando sangramento local e circunferencial ao foco ${ }^{10}$.

A hemorragia tem como principal causa vasculopatias. Em cães, vasculopatias por malformações arteriovenosas e aterosclerose são raras, tendo uma ocorrência maior por coagulopatias e êmbolos sépticos, neoplásicos. Alguns autores descreveram casos individuais de infarto cerebral decorrente de aterosclerose, malformação arteriovenosa ou afecção tromboembólica de origem indeterminada. O hipotiroidismo é a causa possível de aterosclerose em cães ${ }^{16}$.

O cérebro depende em grande parte do metabolismo oxidativo da glicose, para que sejam atendidas suas necessidades energéticas. Para isso o fluxo sanguíneo cerebral deve ser mantido constante, sendo determinado pela pressão de perfusão cerebral. A resposta do tecido nervoso a enfermidades são muito limitados, quase todas as afecções causam gliose, proliferação e congestão dos vasos, e pelo menos, algum edema. A necrose cerebral é liquefativa e pode ser focal ou difusa, sendo o amolecimento do cérebro seguido de necrose denominado de encefalomalacia ${ }^{16}$.

Quando ocorre um processo isquêmico no cérebro, uma série de fatores levam a morte neuronal. A isquemia, leva uma diminuição nos níveis energéticos teciduais, conseqüentemente as bombas de membrana não conseguem manter a homeostasia iônica, que forma um edema intracelular, ocasionando insuficiência elétrica, ocorrendo deslocamento de neurotransmissores e de cálcio, levando a uma ativação excessiva de enzimas, que leva a peroxidação de membranas lipídicas, com produção de níveis tóxicos de ácido araquidônico, prostaglandinas, leucotrienos e radicais livres. O momento exato da ocorrência de danos irreversíveis depende do tipo de neurônio ${ }^{3}$.

Quando ocorre todo este processo, há um deslocamento de água no espaço extracelular para o intracelular, levando ao edema, que na presença deste os dendritos aumentam de volume, a neurotransmissão é interrompida e ocorre sintomas neurológicos ${ }^{10}$. 
VALENTE, D.P. et al. Acidente vascular cerebral em cão - Relato de caso. PUBVET, Londrina, V. 6, N. 25, Ed. 212, Art. 1412, 2012.

A avaliação clínica do paciente é de fundamental importância, e deve ser orientada por distúrbios que interrompem o suprimento de substrato a todo cérebro, ou interferem no mesmo, com arritmias cardíacas, anemias, hipoglicemia e patologias pulmonares, renais e hepáticas em fase terminal 3,10 .

O AVC hemorrágico já foi citado em associação com a ruptura vascular congênita, tumor cerebral primário e secundário, linfoma intravascular, angiopatia amilóide cerebral e doença inflamatória de veias e artérias. Quando este processo ocorre o sangue vai diretamente para o cérebro formando um hematoma no parênquima cerebral, ou no espaço subaracnóideo. A massa do sangue coagulado provoca lesões físicas do tecido, levando a uma alteração no equilíbrio entre volume e pressão, o que leva a um aumento da pressão intracraniana e redução do fluido cérebroespinhal ${ }^{10}$.

Como forma de diagnóstico, devemos excluir outras formas de encefalopatias que possuem características semelhantes já citadas acima 4,13. Em geral, são indicados os seguintes procedimentos médicosveterinários: exames de sangue e urina, para identificar as causas subjacentes possíveis; medição da pressão sanguínea sistólica, para descartar hipertensão; realização de eletrocardiogramas; análises de fezes e culturas de sangue, para descartar a possibilidade de infestações parasitárias; e a análise líquida cérebroespinal, que pode ajudar a descartar doenças inflamatórias do sistema nervoso central, e ainda revelar hemorragias recentes ${ }^{10,11}$.

Os exames que geram imagens em alta definição - conhecidos por tomografia computadorizada (TC) e ressonância magnética (RM) - podem revelar lesões focais. Porém, nem sempre é fácil diferenciar tais lesões das de outras doenças como, por exemplo, a neoplasia ${ }^{4,13}$.

$\mathrm{O}$ alto custo dos exames de imagem e a existência de poucos centros especializados em exames em animais são os principais obstáculos enfrentados no diagnóstico de AVC em animais. Outra dificuldade é que, 
VALENTE, D.P. et al. Acidente vascular cerebral em cão - Relato de caso. PUBVET, Londrina, V. 6, N. 25, Ed. 212, Art. 1412, 2012.

diferente das pessoas, os animais não colaboram na realização dos exames, por isso é necessário anestesiar o animal para obter resultados precisos ${ }^{4}$. Já se sabe que hoje, a conscientização desses exames é de alto valor, para que o diagnóstico seja preciso e assim, o tratamento seja feito de forma adequada.

A ocorrência de AVC em cães é um acontecimento raro, sendo assim o tratamento preventivo é de fundamental importância, principalmente em animais senis, uma vez que estes pertencem a uma classe de maior acometimento $^{10}$. O tratamento, nos casos de AVC hemorrágico, é feito através do uso de diuréticos, corticosteróides, que diminuem a inflamação e - edema do SNC, nas convulsões ativas pode-se fazer o uso de anticonvulsivantes, alem de controlar a ingestão de liquidos. No AVC isquêmico, usa-se os vasodilatadores cerebrais, vitaminas do complexo B e também corticóides ${ }^{3,4}$.

\section{Relato de caso:}

No dia 23/04/2011 animal da raça Pastor Alemão, com 14 anos de idade, começou a apresentar quadro de decúbito e redução de apetite, não apresentando vômito nem diarréia.

Animal foi levado ao veterinário que diagnosticou Cinomose a partir da sintomatologia clínica. Médico veterinário prescreveu: Amoxilina, Prednisona, Buscopan e Glicopan, porém o proprietário só administrou no dia 23/03, pois segundo ele o animal não conseguia deglutir a partir do dia $24 / 3$.

Dia 29/03 animal foi levado ao Centro de Estudos em Clínica e Cirurgia de Animais (CECCA -PUC Betim) para nova avaliação. Ao exame clinico: Animal apresentou desidratação acentuada, mucosas normocrômicas, temperatura de $38,6{ }^{\circ} \mathrm{C}$, taquicardia, estertor úmido, presença de secreção bilateral nas narinas, melena na região perineal, caquexia, hiperextensão de membros anteriores, reflexo biceptal e triceptal aumentados, reflexo de VII 
VALENTE, D.P. et al. Acidente vascular cerebral em cão - Relato de caso. PUBVET, Londrina, V. 6, N. 25, Ed. 212, Art. 1412, 2012.

par de nervos craniano, animal em estado semi-comatoso, mantido em decúbito lateral.

A partir dos sinais clínicos foram sugeridas três suspeitas: Tumor Cerebral, Acidente Vascular Cerebral, Cinomose.

Proprietário optou pela eutanásia, sendo sugerido pelo veterinário a necropsia. Esta foi realizada no laboratório de anatomia patológica do CECCA PUC- Betim; Aos achados macroscópicos: Animal em rigor mortis completo em bom estado de conservação. Emaciação intensa, mucosas externas discretamente hipocoradas, presença de quantidade moderada de secreção nasal muco purulenta com hiperemia das conchas nasais. Linfonodos superficiais moderadamente aumentados de tamanho, com presença de hiperplasia linfóide. Baço aumentado de tamanho, com presença de múltiplos nódulos de coloração avermelhada salientes à superfície, variando de aproximadamente 0,5 a $2 \mathrm{~cm}$ de diâmetro. Pulmões congestos, de coloração avermelhada bilateral, pesados hipocrepitantes, pouco colabados à abertura do tórax e com presença de quantidade moderada de espuma brancacenta no lúmen dos brônquios e da traquéia (edema pulmonar). Presença de grande coágulo, de aproximadamente 3,5 $\mathrm{cm}$ de diâmetro, localizado na região ventrículo lateral esquerdo do encéfalo,na região do corpo caloso, insinuando-se em parto para o lado direito, formando área cavitária nos tecidos ao redor, com suspeita de ruptura de aneurisma, que não pode ser facilmente identificado na necropsia. Glândulas adrenais acentuadamente aumentadas de tamanho, com aumento da cortical e da medular (hiperplasia), com provável quadro de hiperadrenocorticismo.

\section{Discussão:}

A princípio ocorreu a suspeita de cinomose, devido os sintomas apresentados, como secreção bilateral nas narinas, melena na região 
VALENTE, D.P. et al. Acidente vascular cerebral em cão - Relato de caso. PUBVET, Londrina, V. 6, N. 25, Ed. 212, Art. 1412, 2012.

perianal, e as alterações neurológicas, como o delírio, alterações motoras e também por ser um animal senil ${ }^{15}$.

Existiu também a suspeita de tumor cerebral, devido este poder comprimir o parênquima cerebral, levando a uma isquemia, provocando lesão neurológica ${ }^{9}$.

Através da necropsia foi excluído a suspeita de tumor, já que não foi encontrado nenhum tumor, e o cérebro do animal apresentava um coágulo indicando que ocorreu uma hemorragia cerebral, já de cinomose não se excluiu a suspeita, por falta de material para exames. ${ }^{9,15}$.

Além dos achados de necropsia na região encefálica, no corpo caloso, foi encontrado a glândula adrenal aumentada, ou seja indicativo de hiperadrenocorticismo que é uma doença comum em cães; em animais com insuficiência da adrenal, o músculo liso vascular perde a capacidade de resposta a noroepinefrina e epinefrina, com isso os capilares se dilatam tornando-se permeáveis a corantes coloidais. A incapacidade de responder a noraepinefrina nas terminações nervosas prejudica a compensação vascular para a hipovolêmica da insuficiência adrenal promovendo assim o colapso vascular, o que levaria a uma diminuição da resistência dos vasos sanguíneos, ocasionando uma hemorragia ${ }^{5,6}$.0 hiperadrenocorticismo justificaria as áreas hemorrágicas no baço e na bexiga, por causar essa deficiência na coagulação.

O diagnóstico foi dado através da necropsia, mas pode ser feito para melhor confirmação ante-mortem, tomografia computadorizada e ressonância magnética, para definir a região vascular envolvida determinando a extensão da lesão, para haver distinção entre AVC isquêmico e AVC hemorrágico. A histopatologia pode ser usada post-mortem para definir a causa ${ }^{3,10}$.

Para ter uma melhor estabilidade do paciente e proteção da área envolvida , deve haver monitoramento dos sinais vitais como status neurológico, identificar e tratar causas latentes. O risco de ocorrer um deterioração neurológica e cardiovascular é alta nas primeiras 24 horas da lesão, já o 
VALENTE, D.P. et al. Acidente vascular cerebral em cão - Relato de caso. PUBVET, Londrina, V. 6, N. 25, Ed. 212, Art. 1412, 2012.

desenvolvimento do edema vascular ocorre em tempo mais lento. Um monitoramento cuidadoso é essencial durante o início do período dos parâmetros vitais para um bom estado neurológico ${ }^{10}$.

Para confirmar o diagnóstico de AVC é necessário fazer uma histopatologia, observando as áreas afetadas e suas determinadas lesões ${ }^{10}$.

O tratamento é muito variado e dependerá do tipo de AVC, da severidade da disfunção neural. Centram-se assim na prevenção de danos cerebrais secundários ou de complicações, tais como aumento da pressão intracraniana ou apreensões, e identificar e tratar as causas primarias ${ }^{11,16}$. É preciso em primeiro lugar ter um tratamento preventivo, que inclui a identificação e controle de fatores de risco e o uso da terapia antitrombótica. A avaliação e o acompanhamento neurológico regulares são componentes importantes do tratamento preventivo. O controle da hipertensão e da diabetes, o uso de drogas antiplaquetárias ou de anticoagulação têm reduzido a incidência de acidentes vasculares ${ }^{10}$. Caso ocorra o AVC os pacientes devem ser enviados a um serviço de emergência, onde a atenção deve ser dirigida inicialmente para avaliação e controle da respiração e da função cardíaca. Finalmente, o tratamento do paciente com acidente vascular cerebral completo envolve 0 uso de terapêutica antitrombótica, evitando outros acidentes vasculares, e em casos apropriados o uso da cirurgia ${ }^{3,10,11}$.

Com o uso de fisioterapia dentro de alguns dias dentro de um acidente vascular completo, previne complicações, ajuda a evitar rigidez articular e contraturas, ocupando o paciente no processo de recuperação funcional do próprio corpo ${ }^{3}$.

\section{Conclusão:}

O AVC em cães é raro, sendo preciso recomendar e conscientizar sempre os proprietários da importância de um diagnóstico preciso. No presente trabalho, através de necropsia foi possível obter um diagnóstico concreto de 
VALENTE, D.P. et al. Acidente vascular cerebral em cão - Relato de caso. PUBVET, Londrina, V. 6, N. 25, Ed. 212, Art. 1412, 2012.

\author{
AVC, não sendo possível, através desse método, definir a causa. Para isso, \\ seria necessário fazer histopatologia.
}

\title{
Referências:
}

1. BRUCHIM, Y.; KLEMENT, E.; SARAGUSTY, J.; FINKEILSTEIN, E.; KASS, P.; AROCH, I.: Heat stroke in dogs: A retrospective study of 54 cases (1999-2004) and analysis of risk factors for death. J Vet Inter. v.20, n.1, p. 38-46. 2006.

2. COELHO, R. S.; FREITAS, W. M.; CAMPOS, G. P.; TEIXEIRA, R. A.: Stroke awareness among cardiovascular disease patients. Arg Neuropsiquiatr, v. 66, n. 2, p. 209-212, 2008.

3. COLLINS, Robert C. Doenças vasculares cerebrais. In: COLLINS, Robert C. Neurologia. Rio de Janeiro: Guanabara Koogan S.A, 1998. p.171-182.

4. CERVERA V.; MAI, W.; VITE, CH.; JOHNSON, V.; DAYRELL-HART. B.; SEILER, G.S.: Comparative magnetic resonance imaging findings between gliomas and presumed cerebrovascular accidents in dogs. Vet Radiol Ultrassound. v. 52, n.1, p. 33-40. 2011.

5. GANONG, W. F. A medula adrenal e o córtex adrenal. In: GANONG, W. F. Fisiologia Medica.Rio de Janeiro:Prentice-Hall do Brasil,1998. p. 262.

6. JONES, T. C. Glândulas Endócrinas. In: JONES, T. C. Patologia Veterinária. São Paulo: Manole LTDA, 2000. p. 1269- 1271.

7. GAROSI, L.S., MCCONNELL J.F.: Ischaemic stroke in dogs and humans: a comparative review. J Small Anim Pract. v.46, n.11, p. 521-529. 2005.

8. GAROSI, L.S.: Cerebrovascular disease in dogs and cats. Vet Clin North Am Small Anim Pract. v.40, n.1, p.65-79. 2010.

9. GALLO, P.: Hemorrhage in cerebral metastasis from angiosarcoma of the heart: case report. Arq. Neuro-Psiquiatr., São Paulo, v. 59, n. 3B, set. 2001.

10. GAROSI, L. S.: Cerebrovascular disease in dogs and cats. Veterinary Clinics of North America: Small Animal Practice, v. 40, n. 1, p. 65-79, 2010.

11. HUNNING, P. S.; RIGON, G. M.; AGUIAR, J.; STEDILE, R.; COLOME, L. M.: Sindrome do cão tremedor. Acta Scientiae Veterinariae. v. 38 n. 2, p. 209-212. 2010.

12. MACRAE, I.: Pre-clinical stroke research -advantages and disadvantages of the most common rodent models of focal ischaemia. Br J Pharmacol. 2011.

13.PAUL, A.E.; LENARD, Z.; MANSFIELD, C.S.: Computed tomography diagnosis of eight dogs with brain infarction. Aust Vet J. v.88, n.10, p. 374-80. 2010.

14.RICHARD, M.J.; CONNELL, B.J.; KHAN, B.V.; SALEH, T.M.: Cellular mechanisms by which lipoic acid confers protection during the early stages of cerebral ischemia: a possible role for calcium. Neurosci Res. v.69, n.4, p.299-307. 2011.

15. SILVA, M. C.: Neuropatologia da cinomose canina: 70 casos (2005-2008). Pesq. Vet. Bras., Rio de Janeiro, v. 29, n. 8, ago. 2009.

16. KORNEGAY, J. N. Patogênese das afecções do sistema nervoso central. In: SLATTER, D. Manual de cirurgia de pequenos animais. São Paulo: Manole Ltda, 1988. p. 1231 1240. 
17.TARDINI, D. M. S.; YOSHIDA, W. B.; NOVELLI, E. L. B.; SEQUEIRA, J. L. Avaliação de dois modelos experimentais de isquemia e reperfusão cerebral em ratos com oclusão temporária carotídea associada ou não à oclusão vertebral. Acta Cir. Bras. v. 18, n. 6, p. 514-517. 2003. 